\title{
Non-Orthogonal Waveform Scheduling for Next Generation Narrowband IoT
}

\author{
Tongyang $\mathrm{Xu}$ \\ Department of Electronic and \\ Electrical Engineering \\ University College London \\ Email: tongyang.xu.11@ucl.ac.uk
}

\author{
Izzat Darwazeh \\ Department of Electronic and \\ Electrical Engineering \\ University College London \\ Email: i.darwazeh@ucl.ac.uk
}

\begin{abstract}
Narrowband Internet of Things (NB-IoT), firstly introduced in 3GPP release 13, brings benefits such as low power and wide coverage. Repetitive transmission is the mechanism in uplink channels in NB-IoT for coverage extension. This is done at the expense of extra power consumption and time latency. In this work, we propose a power efficient framework termed enhanced NB-IoT (eNB-IoT), which adopts a non-orthogonal waveform that can bring additional power budget without using the repetitive transmission mechanism. The waveform halves the required bandwidth, for a given bit rate, whilst maintaining the same BER performance. We propose to divide the frequency band into a set of sub-bands and then apply adaptive waveform scheduling to the modulated data on the optimal frequency band with lower loss and therefore higher signal-to-noise ratio (SNR). Advantageously, the narrower bandwidth of fast-orthogonal frequency division multiplexing (Fast-OFDM) waveform can flexibly map the signal on the frequency bands associated with high SNR and avoid transmission in the higher loss frequency band. Hence, compared to the single-carrier-frequency division multiple access (SC-FDMA) signal, which is the default uplink signal format of NB-IoT, and which occupies all the signal bandwidth in bands of high and low SNR, simulation results show that $\sim 84 \%$ power can be saved via using the Fast-OFDM signal in a defined nonline-of-sight channel. Experimental results show a $\sim 11 \mathrm{~dB}$ error vector magnitude (EVM) improvement compared to the SCFDMA signal. The saved power can extend signal coverage where repetitive transmission is limited.

Index Terms-Internet of Things (IoT), narrowband, NB-IoT, enhanced NB-IoT (eNB-IoT), 5G, non-orthogonal, power efficiency, spectral efficiency, waveform, scheduling, software defined radio, experiment, testbed, coverage extension.
\end{abstract}

\section{INTRODUCTION}

Internet of Things (IoT) require low power consumption, long range communication and massive connected devices. Narrowband is the basic signal format used in different IoT standards such as LoRa [1], SigFox [2] and NB-IoT [3]. The first two techniques are used in free frequency bands. NB-IoT [3] was proposed with security promise occupying licensed frequency band. One physical resource block (PRB) within long term evolution (LTE) is allocated to NB-IoT. Thus the signal is modulated on 12 sub-carriers with bandwidth of 180 $\mathrm{kHz}$. Since NB-IoT follows the conventional LTE standard, the deployment is straightforward.

As predicted in [4], the number of IoT devices would reach billions by 2020 . Therefore, each IoT device has to be made as cheap as possible to enable massive devices connection. There are many existing options, described in [5], to meet the low complexity requirement such as limited bandwidth, reduced data rate, single antenna and half duplex protocol. Additionally, the simplified hardware design would lengthen battery life of each IoT device. The research on power efficiency improvement has been studied in work [6]. Two power saving schemes such as power save mode (PSM) and extended discontinuous receive (eDRx) are introduced in [6]. To achieve deep indoor coverage, advanced techniques listed in [5] such as repetitive transmission, power spectral density (PSD) boosting, single-tone uplink transmission and low order modulation schemes are proposed for NB-IoT.

In order to get benefits from frequency diversity, NB-IoT introduced single-tone frequency hopping [7], [8] for uplink channels associated with the repetitive transmission. However, in addition to the time delay introduced by repetitive transmission, the frequency hopping of NB-IoT has some other challenges. At the receiver, the resource elements (i.e. tones) of different hops would be combined to form one complete PRB. However, as explained in [7], limitations exist for this scheme. First, when combining different resource elements, a residual clock offset would result in ranging errors. Second, retuning of the RF front-end to each narrowband hop would introduce a random frequency offset. Third, the hops introduce time delay and would be beyond coherence time, thus the number of hops is limited. Last, the sparse frequency allocation of each resource element would cause interference due to the sidelobes of employed signal waveform. Therefore, a solution, which can avoid single-tone frequency hopping and redundant repetitive transmission, would significantly improve performance. In this work, we propose an adaptive non-orthogonal waveform scheduling mechanism for power efficiency improvement in the next generation NB-IoT. Typical NB-IoT devices employ SC-FDMA signals for the uplink channel and the signal occupies fixed band portions within LTE. However, the signal may experience both good quality and poor quality channels. Fast-OFDM was firstly proposed in 2002 [9] and it has been practically used in IoT communications in [10], in which it proves that by using bandwidth compressed waveforms, the number of IoT devices can be doubled. However, no waveform scheduling was used and power efficiency was not considered. Since the Fast-OFDM compresses $50 \%$ of bandwidth with no 
performance loss, the aim of this work is to locate optimally the Fast-OFDM signal of $90 \mathrm{kHz}$ bandwidth within the $180 \mathrm{kHz}$ frequency band according to the feedback SNR. The principle is to compute channel state information (CSI) (indicates SNR) on each sub-carrier and the half bandwidth Fast-OFDM signals will be shifted to the optimal frequency band associated with the highest CSI. Simulation results in this work show that in order to achieve the same BER performance, the use of Fast-OFDM in enhanced NB-IoT (eNB-IoT) [10]-[12] saves transmission power by $\sim 84 \%$. Experimental results show that in the same channel condition, Fast-OFDM waveform can be allocated to an optimal frequency portion leading to an improved EVM by $\sim 11 \mathrm{~dB}$. In summary, the improved power efficiency by using the Fast-OFDM waveform scheduling brings benefits such as extending IoT signal coverage where the repetitive transmission is limited

\section{WAVEFORM COMPRESSION}

The sub-carrier packing schemes of OFDM and Fast-OFDM are shown in Fig. 1 where both signals occupy 12 sub-carriers. It is clearly shown that the benefit of Fast-OFDM is its closer sub-carrier packing spacing and therefore compressed bandwidth.

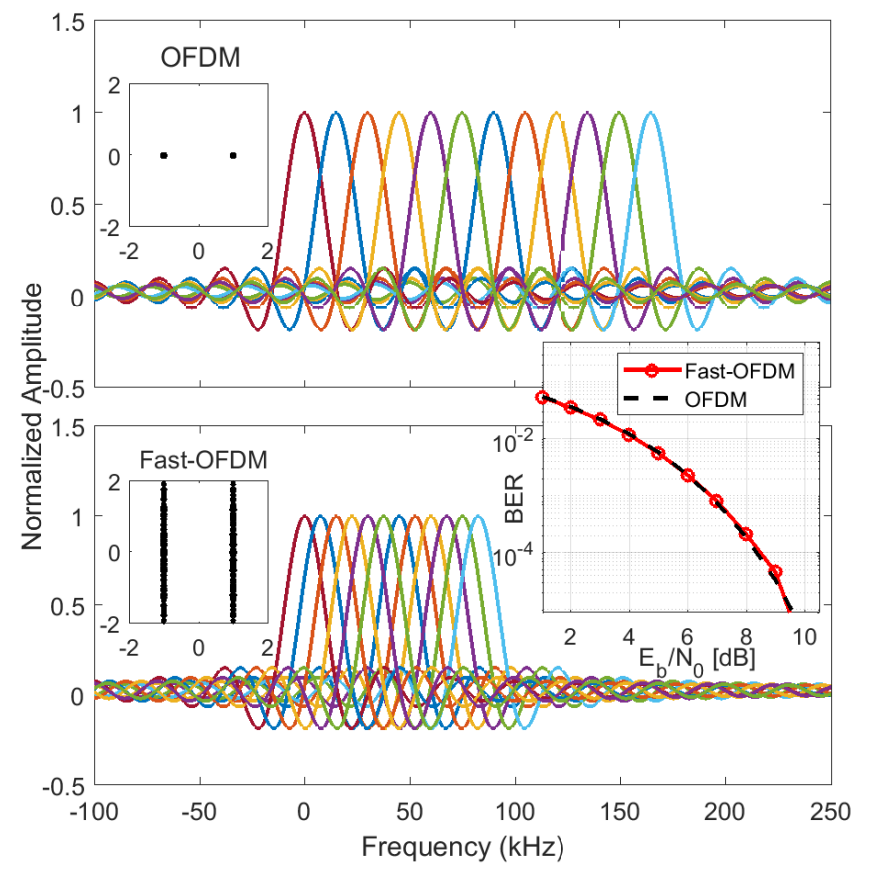

Fig. 1. Sub-carrier allocation schemes, constellations and BER for OFDM and Fast-OFDM. BPSK is used in this comparison.

Work in [9] explains that although sub-carriers are packed closer violating orthogonality, inter carrier interference (ICI) can be perfectly eliminated for Fast-OFDM at the cost of using one-dimensional modulation scheme such as binary phase shift keying (BPSK). The reason is in that only the real part of a Fast-OFDM signal has frequency orthogonality features whilst its imaginary part is non-orthogonal and therefore will suffer ICI, leading to the unusual constellation pattern for Fast-OFDM, which is shown in Fig. 1. An ideal Fast-OFDM constellation is presented where the real part shows perfect ' +1 ' and ' -1 ' (on the I-axis) while its imaginary part is greatly interfered with amplitude ranging from ' -2 ' to ' +2 ' (on the Q-axis). Therefore, only the real part can be effectively used. Its BER performance is studied and also illustrated in Fig. 1. Although imaginary part introduces interference, its real part can achieve the same performance as OFDM. The mathematical format of Fast-OFDM signal is expressed as

$$
x(t)=\sum_{k=0}^{N-1} X[k] e^{j 2 \pi \frac{k}{2 T} t}
$$

where $N$ is the number of sub-carriers and $X[k]$ is onedimensional modulation symbols such as BPSK. The subcarrier spacing is defined as $\frac{1}{2 T}$ where $T$ is one Fast-OFDM symbol period and the frequency for each sub-carrier is defined as $\frac{k}{2 T}$. In order to define the interference within Fast-OFDM, correlation of two symbols is studied in the following.

$$
\begin{aligned}
\operatorname{corr}(m, n)= & \frac{1}{T} \int_{0}^{T} X[m] X[n]\left(e^{j 2 \pi \frac{m}{2 T} t}\right)\left(e^{j 2 \pi \frac{n}{2 T} t}\right)^{*} d t \\
= & X[m] X[n]\{\operatorname{sinc}[\pi(m-n)] \\
& \left.+j \cdot \frac{\pi(m-n)}{2} \cdot \operatorname{sinc}^{2}\left[\frac{\pi(m-n)}{2}\right]\right\}
\end{aligned}
$$

where its real and imaginary parts are shown in (3) and (4), respectively.

$$
\begin{gathered}
\Re\{\operatorname{corr}(m, n)\}= \begin{cases}X[m] X[n] & m=n \\
0 & m \neq n\end{cases} \\
\Im\{\operatorname{corr}(m, n)\}= \begin{cases}0 & m=n \\
\frac{\pi(m-n)}{2} \cdot \operatorname{sinc}^{2}\left[\frac{\pi(m-n)}{2}\right] & m \neq n\end{cases}
\end{gathered}
$$

It is observed that for the real part signal cross correlation elements $(m \neq n)$ are zeros indicating no interference among sub-carriers. However, the imaginary part of the signal shows non-zero cross correlation. This mathematically explains that BPSK modulated Fast-OFDM signals can be recovered even with its self-created ICI.

\section{WAVEFORM SCHEDULING}

Conventional scheduling in NB-IoT, such as signal repetition, is dependent on signal coverage. In weak signal coverage scenarios, signal quality is enhanced by transmitting multiple copies of the same signal at the cost of longer transmission time and possible frequency offset. In this section, we consider a non-orthogonal waveform frequency re-selection scheme, which can boost the performance and reduce the number of signal repetition.

In non-line-of-sight channels, due to frequency selective characteristics, the receiver SNR values are variant at different frequencies. The narrower signal bandwidth of Fast-OFDM can potentially avoid poor quality channels and therefore brings 
higher SNR and better BER performance. Channel conditions indicated by SNR can be calculated at the base station and the control information, including the calculated SNR, is sent to IoT devices for waveform frequency re-selection. Thus, IoT devices can select their preferred channel frequency portions associated with high SNR. In this case, Fast-OFDM based eNB-IoT signals can work at their best frequencies and reduce the probability of occupying poor conditioned channels.

In order to evaluate the benefits of using the bandwidth compressed waveform in wireless channels, a static frequency selective channel [13] is tested and mathematically expressed in (5) as an example.

$$
\begin{aligned}
h(t)= & 0.8765 \delta(t)-0.2279 \delta\left(t-T_{s}\right) \\
& +0.1315 \delta\left(t-4 T_{s}\right)-0.4032 e^{\frac{j \pi}{2}} \delta\left(t-7 T_{s}\right)
\end{aligned}
$$

The channel has four paths and the maximum time delay is seven times longer than the sample period $T_{s}$. Thus, the frequency response is no longer flat and the channel is frequency selective. The spectrum of NB-IoT signals passing through this channel is shown in Fig. 2(a) where the signal experiences high power loss (i.e. low SNR) at high frequency. This results in performance degradation to signals especially SC-FDMA due to its wide bandwidth of $180 \mathrm{kHz}$. Fortunately, since the Fast-OFDM signal compresses bandwidth to $90 \mathrm{kHz}$, it can avoid the significant power loss at high frequency as shown in Fig. 2(b). Therefore, the BER performance of FastOFDM is expected to be better than that of SC-FDMA. For the purpose of simplification, instead of calculating SNR, we use CSI magnitude as the channel condition indicator. The normalized CSI magnitude on each sub-carrier, distorted by the channel defined in (5), is calculated and illustrated in Fig. 3.

The principle of the adaptive waveform scheduling is to find eNB-IoT optimal frequency portions of a total $90 \mathrm{kHz}$ bandwidth within the limited $180 \mathrm{kHz}$ NB-IoT bandwidth. First, an IoT device sends a training signal of $180 \mathrm{kHz}$ to a base station via uplink channels. The base station estimates the channel condition of the $180 \mathrm{kHz}$ bandwidth signal. Then the optimal $90 \mathrm{kHz}$ frequency band portion is determined based on the highest CSI magnitude. The frequency adaptation information is sent back to the IoT device and the eNB-IoT signal is adaptively shifted to the best channel portion. In a realistic uplink signal time-frequency structure design, the training signal has to be designed carefully considering channel coherence time. One design option is shown in Fig. 4. The first demodulation reference signal (DMRS), performing as the training signal, is used for the waveform scheduling and the second DMRS is for Fast-OFDM signal channel estimation. In this work, the waveform scheduling DMRS is sent in each subframe. However, the time-frequency structure can be flexibly designed according to channel characteristics.

In detail, we estimate CSI on 12 orthogonal NB-IoT subcarriers based on the waveform scheduling DMRS. Then, six consecutive sub-carriers with the highest CSI magnitudes are searched. The reason for the six CSI coefficient values is that

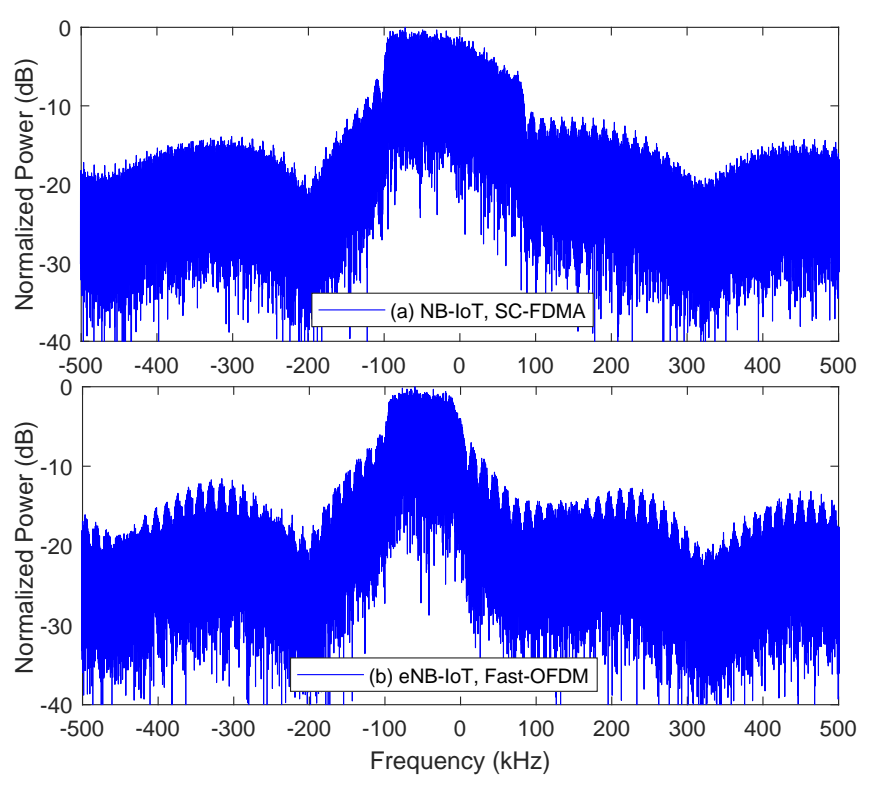

Fig. 2. Spectra comparisons of typical NB-IoT (SC-FDMA signal) and eNB-IoT (Fast-OFDM signal) in the defined frequency selective channel. The location of the NB-IoT signal is fixed between $-90 \mathrm{kHz}$ and $90 \mathrm{kHz}$ indicating a bandwidth limited scenario. The eNB-IoT signal can select the optimal frequency portion occupying the regime between $-90 \mathrm{kHz}$ and $0 \mathrm{kHz}$ due to the bandwidth compression.

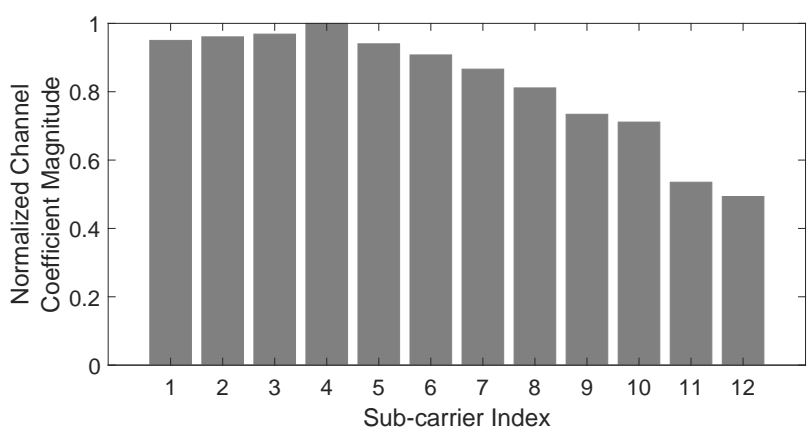

Fig. 3. Normalized channel coefficient magnitude on 12 sub-carriers within $180 \mathrm{kHz}$ NB-IoT frequency band.

Fast-OFDM occupies half of the bandwidth of SC-FDMA. Thus, a sliding window is introduced to find the optimal CSI coefficient combinations. The operation is to average the first six coefficient values and then move to the next six values with one sub-carrier spacing as one sliding step. Thus the sliding window operation is expressed as

$$
C S I_{\text {mean }}(k)=\frac{1}{6} \sum_{i=k}^{k+5} C S I_{\text {sub }}(i)
$$

where $C S I_{\text {mean }}(k)$, with index $k=0,1, \ldots, 6$, is the average of six $C S I_{\text {sub }}$. Therefore, seven $C S I_{\text {mean }}$ values are obtained and the optimal frequency band is selected associated with the maximum $C S I_{\text {mean }}$. 


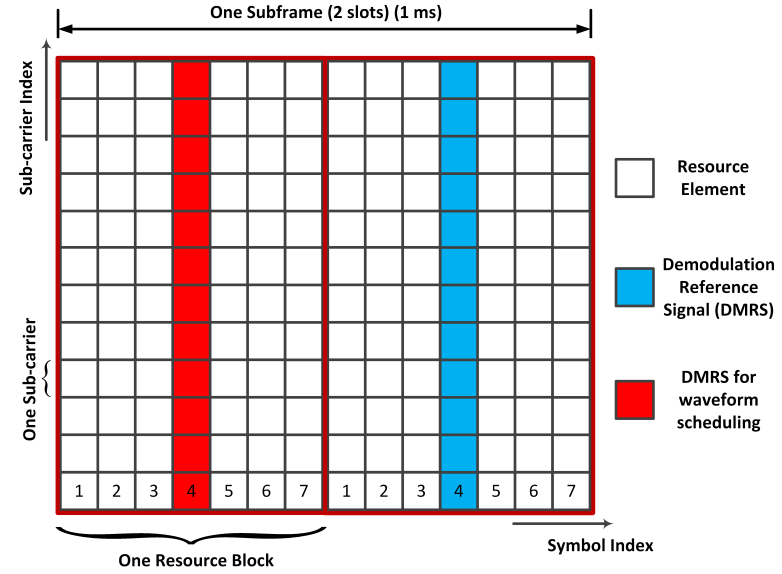

Fig. 4. eNB-IoT uplink time-frequency resource block definition for a single antenna system.

In a bandwidth limited scenario such as $180 \mathrm{kHz}$, the typical NB-IoT signals cannot realize the waveform scheduling. However, for eNB-IoT, due to the bandwidth compression, the saved spectrum can be reserved for signal band shifting. The proposed non-orthogonal waveform scheduling in eNBIoT is more efficient than the frequency hopping in NB-IoT since challenges such as coherence time violation, frequency offset, extra power consumption and repetitive transmission time delay do not exist in eNB-IoT.

\section{Simulation Evaluations and Applications}

The signal configurations for both SC-FDMA and FastOFDM are shown in Table I. In this work, Turbo coding, standardized in LTE [14], is applied to mitigate the channel effects. The data is Turbo encoded using a code rate $R_{\text {code }}=1 / 3$. The code used is a $(13,15)$ code of memory 3 with feedforward polynomial $G_{1}(D)=1+D+D^{3}$ and feedback polynomial $G_{2}(D)=1+D+D^{2}+D^{3}$.

Table I: Simulation Specifications

\begin{tabular}{lll}
\hline Parameters & SC-FDMA & Fast-OFDM \\
\hline \hline Center frequency (GHz) & 2.4 & 2.4 \\
\hline Occupied channel bandwidth (kHz) & 180 & 90 \\
\hline Bit rate (kbit/s) & 180 & 180 \\
\hline Bit rate per sub-carrier (kbit/s) & 15 & 15 \\
\hline Sub-carrier bandwidth (kHz) & 15 & 15 \\
\hline Sub-carrier spacing (kHz) & 15 & 7.5 \\
\hline Sampling frequency (MHz) & 1.92 & 1.92 \\
\hline FFT size & 128 & 128 \\
\hline Number of cyclic prefix samples & 10 & 10 \\
\hline Number of guard band sub-carriers & 58 & 58 \\
\hline Number of data sub-carriers & 12 & 12 \\
\hline Modulation scheme & $\pi / 2$-BPSK & BPSK \\
\hline
\end{tabular}

BER comparisons of the two signals are shown in Fig. 5. It is clearly seen that the BPSK modulated Fast-OFDM has better performance than the $\pi / 2$-BPSK modulated SC-FDMA at different BER levels. The reason for this is due to the Fast-OFDM spectrum compression shown in Fig. 2(b). Both

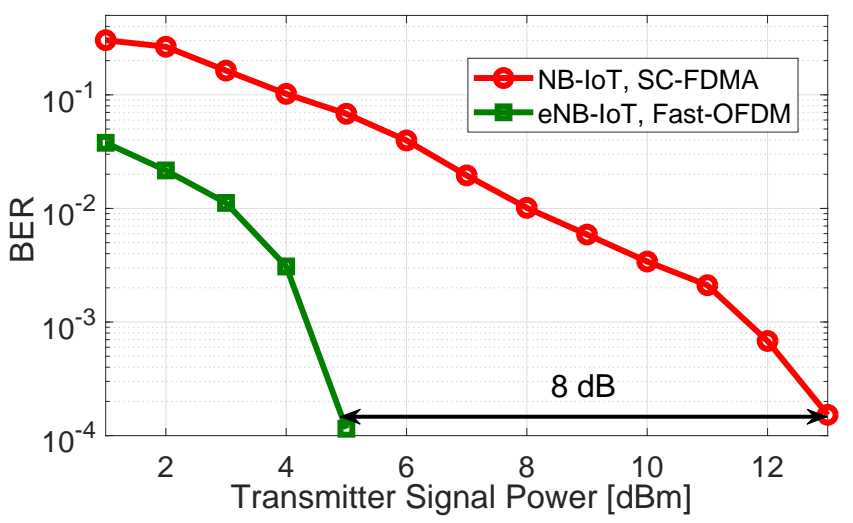

Fig. 5. BER performance versus transmitter signal power of SCFDMA and Fast-OFDM signals in the defined frequency selective channel. The noise power is normalized to be $1 \mathrm{dBm}$.

signals experience the same frequency selective channel in (5). However, since Fast-OFDM compresses bandwidth, it can avoid the significant power loss at high frequency. All the subcarriers of Fast-OFDM are within high SNR regime, therefore, its BER performance is improved. It is shown that with the improvement of BER, the required transmitter power for both Fast-OFDM and SC-FDMA signals is increased. However, the Fast-OFDM requires less power to reach the same BER level. One example is that when BER $=1.5 \times 10^{-4}$, Fast-OFDM can save approximately $8 \mathrm{~dB}$ transmission power. In terms of peakto-average power ratio (PAPR) performance, its calculation and comparison can be found in work [15].

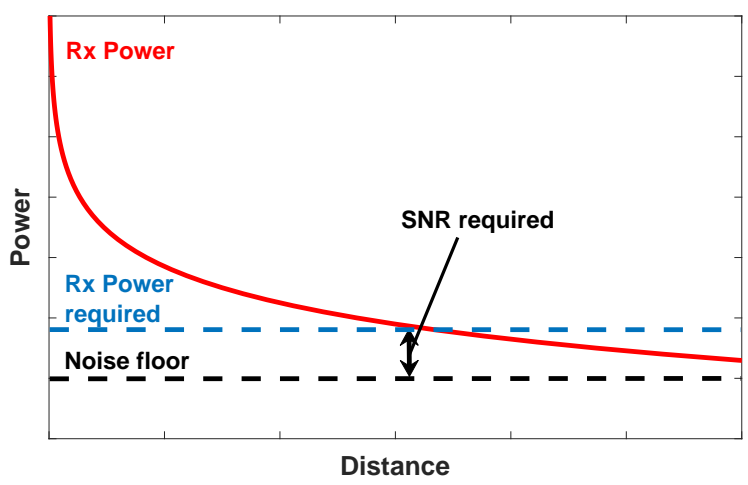

Fig. 6. Illustration of the correlation of received signal power and transmission distance. This indicates that the improved power efficiency by using the non-orthogonal waveform scheduling in eNBIoT can bring benefits such as coverage extension or signal quality improvement.

Fig. 6 shows that with the increase of signal transmission distance, the received signal becomes weak and the power is reduced to a certain level. For a practical wireless communication system, a minimum SNR is required at the receiver in order to recover signals. It is inferred that the additional $8 \mathrm{~dB}$ link budget, derived from Fig. 5, can extend signal coverage 
or enhance signal power in deep indoor scenarios.

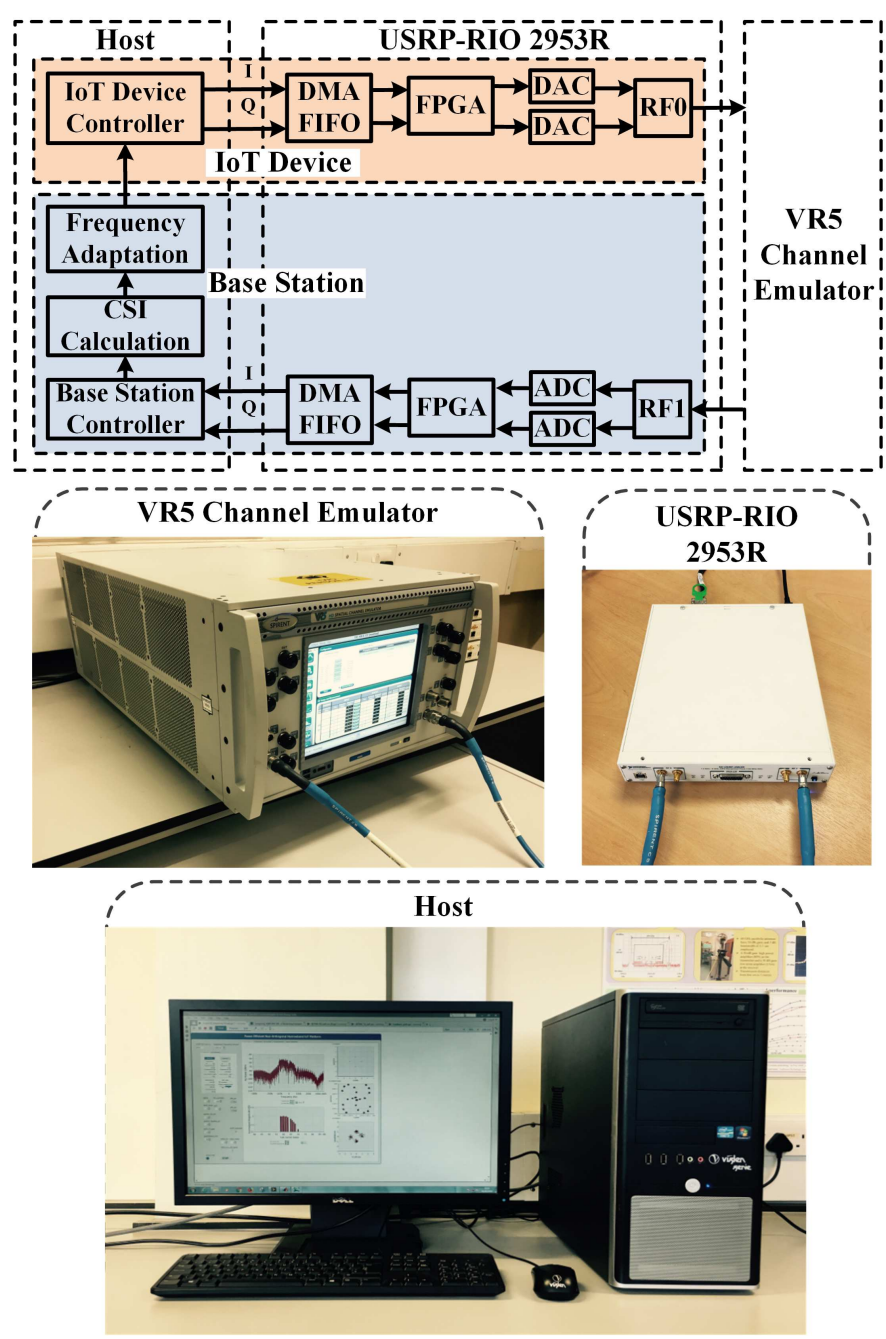

Fig. 7. USRP-RIO based eNB-IoT system implementation block diagram and experiment platform setup.

\section{EXPERIMENT VALIDATION AND RESULTS COMPARISON}

In this section, a practical experiment is designed to verify the performance gains achieved from the proposed nonorthogonal waveform scheduling. In this experiment, we use one universal software radio peripheral (USRP)-RIO 2953R software defined radio (SDR) from National Instruments [16] as a transceiver. The benefit of using this device is its software defined capability using LabVIEW. This fast programmable approach can speed up the experiment design. The experiment platform is set up under the same system specifications in Table I where the fast Fourier transform (FFT) size is set to 128 following the minimum LTE standard and the occupied data sub-carriers is set to 12 following NB-IoT standard [14]. $\pi / 2$ BPSK modulation is for NB-IoT and BPSK for eNB-IoT.

The experiment platform schematic is shown in Fig. 7 where a host, the USRP-RIO 2953R and a VR5 channel emulator are connected together to form a complete system. The connection between the host and the USRP-RIO 2953R is via a MXIExpress $\times 4$ cable, which can support up to $800 \mathrm{MB} / \mathrm{s}$ data streaming and the resolution could reach 16 bits per sample.

Each USRP-RIO has two RF chains, therefore, the upper $\mathrm{RF}$ chain performs as an IoT device and the lower RF chain is operated as a base station. The host is functioned as a controller, which consists of an IoT device controller and a base station controller. The feedback channel is implemented in the host with two functions such as frequency adaptation and CSI calculation.

Table II: Experimental IoT Channel Model

\begin{tabular}{|c|c|c|c|}
\hline Path & Relative Path Loss & Delay Values & Phase Shift \\
\hline 1 & $0 \mathrm{~dB}$ & $0 \mathrm{~ns}$ & 0 \\
\hline 2 & $5.9 \mathrm{~dB}$ & $0.52 \mathrm{~ns}$ & $\pi$ \\
\hline 3 & $8.2 \mathrm{~dB}$ & $2.08 \mathrm{~ns}$ & 0 \\
\hline 4 & $3.4 \mathrm{~dB}$ & $3.64 \mathrm{~ns}$ & $3 \pi / 2$ \\
\hline
\end{tabular}

The IQ data, generated from the IoT device controller, is sent to the USRP-RIO. The IQ data is firstly saved to the direct memory access (DMA) first in first out (FIFO) and then extracted by the Kintex-7 410T FPGA. The integrated FPGA performs functions such as fractional decimator, frequency shift and impairment correction [17]. The processed IQ data is then sent to a digital-to-analogue converter (DAC) and radio frequency (RF) modules. The $\mathrm{RF}$ signal, output from the USRP-RIO, is transmitted via the VR5 channel emulator with the channel defined in Table II. The lower RF chain of USRP-RIO captures the signal and processes the data in the base station where it performs reversed signal processing as the upper RF chain. The IQ data coming from the DMA FIFO via the MXI-Express $\times 4$ cable, is sent to the base station controller for digital signal recovery. After this module, CSI is calculated and the optimal waveform frequency portion is determined. The frequency adaptation information is fed back to the IoT device controller to locate the optimal Fast-OFDM signal band via adaptive frequency shifting. Thus, the signal is optimized based on the feedback CSI. The newly generated IoT signal is sent and received via a closed loop principle.

The experiment results, collected from the USRP-RIO device, are illustrated in Fig. 8 where spectra, channel state information and constellation diagrams are illustrated. The frequency selective channel is defined in the VR5 channel emulator based on the channel model in Table II. Three practical systems are designed and compared. In order to clearly show the channel condition improvement of eNB-IoT, the optimal location of Fast-OFDM based eNB-IoT signal is determined and graphically shown in the figure with the label of "Adaptive Fast-OFDM". SC-FDMA signal modulated with $\pi / 2$-BPSK is the waveform of 3GPP NB-IoT standard [14]. The bandwidth is $180 \mathrm{kHz}$ but with high power distortion at high frequency regime resulting in an EVM of $-10.0 \mathrm{~dB}$. The second signal is the half bandwidth Fast-OFDM waveform, which compresses $50 \%$ of bandwidth and beneficially avoid the high power distortion regime. Thus, its EVM is evidently improved to $-17.7 \mathrm{~dB}$. The adaptive waveform scheduling scheme can flexibly shift the bandwidth compressed Fast- 

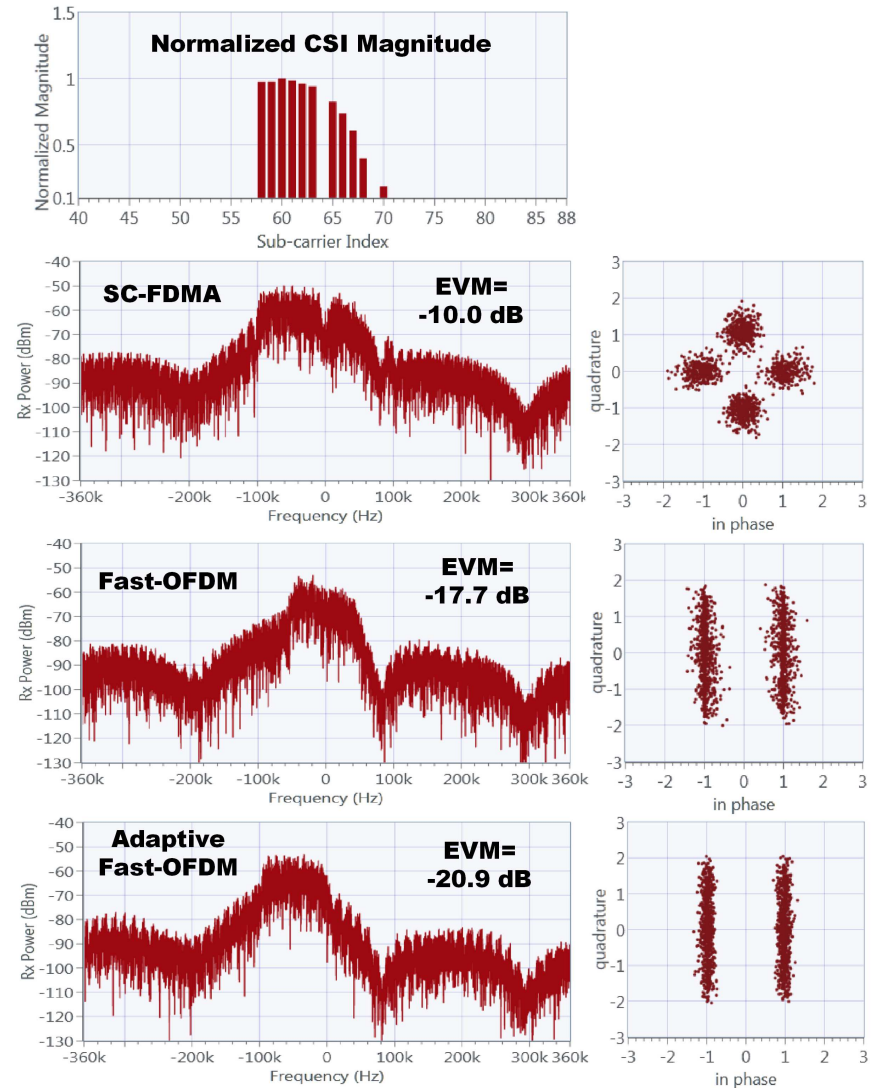

Fig. 8. Experimental Results to show the performance improvement of eNB-IoT relative to NB-IoT. SC-FDMA is used in NB-IoT. FastOFDM and adaptive Fast-OFDM are for eNB-IoT. Data sub-carrier index ranges from 58 to 70 . Basedband spectra are studied with EVM comparisons.

OFDM signal to a frequency portion associated with high SNR. Thus, the performance is further improved with an EVM of -20.9 dB. In summary, the Fast-OFDM based eNBIoT outperforms the SC-FDMA based NB-IoT in frequency selective channels.

\section{CONCLUSIONS}

A non-orthogonal IoT waveform scheduling scheme was proposed and practically tested in this work for the next generation enhanced narrowband IoT (eNB-IoT). The aim is to improve power efficiency of existing NB-IoT via using a nonorthogonal waveform Fast-OFDM. NB-IoT was proposed by 3GPP to support deep coverage using repetitive transmission mechanism at the cost of increased power consumption and time delay. The battery life of each IoT device is limited and saving power for each IoT device is of great importance. FastOFDM has a narrower bandwidth than SC-FDMA leading to an efficient frequency shifting by avoiding the frequency band associated with low SNR while the legacy SC-FDMA based NB-IoT has to cover both high and low SNR frequency portions. We investigated BER performance for both NB-IoT and eNB-IoT in a static frequency selective channel. Their performance comparison is dependent on the channel frequency response portion within which the compressed spectrum of Fast-OFDM is located. Simulation results show that in order to achieve the same BER performance in a static frequency selective channel, Fast-OFDM only requires $\sim 16 \%$ of the SCFDMA signal power (saving $\sim 84 \%$ ). Experiment in this work verifies the feasibility of using the non-orthogonal waveform scheduling for eNB-IoT. Practical results obtained from the software defined radio device USRP-RIO show that the proposed waveform scheduling scheme enables Fast-OFDM signal to achieve a significant $\sim 11 \mathrm{~dB}$ EVM gain than the typical SCFDMA signal in the defined frequency selective channel.

\section{ACKNOWLEDGEMENT}

This work was funded by the Engineering and Physical Sciences Research Council (EPSRC) "Impact Acceleration Discovery to Use" award (Grant reference: EP/K503745/1). This work was also supported by National Instruments.

\section{REFERENCES}

[1] N. Sornin, M. Luis, T. Eirich, T. Kramp, and O.Hersent, "LoRaWAN specification," LoRa Alliance, technical document, Version 1.0, Jan. 2015.

[2] SigFox, "SigFox," https://www.sigfox.com, April 2018.

[3] M. Chen, Y. Miao, Y. Hao, and K. Hwang, "Narrow band Internet of Things," IEEE Access, vol. 5, pp. 20 557-20 577, 2017.

[4] Ericsson, "More than 50 billion connected devices," Ericsson, White paper, Feb. 2011.

[5] Qualcomm, "Paving the path to narrowband 5G with LTE Internet of Things (IoT)," Qualcomm Technologies, Inc, White paper, Jul. 2016.

[6] A. Rico-Alvarino, M. Vajapeyam, H. Xu, X. Wang, Y. Blankenship, J. Bergman, T. Tirronen, and E. Yavuz, "An overview of 3GPP enhancements on machine to machine communications," IEEE Communications Magazine, vol. 54, no. 6, pp. 14-21, June 2016.

[7] J. A. del Peral-Rosado, J. A. Lpez-Salcedo, and G. Seco-Granados, "Impact of frequency-hopping NB-IoT positioning in 4G and future 5G networks," in 2017 IEEE International Conference on Communications Workshops (ICC Workshops), May 2017, pp. 815-820.

[8] Y. P. E. Wang, X. Lin, A. Adhikary, A. Grovlen, Y. Sui, Y. Blankenship, J. Bergman, and H. S. Razaghi, "A primer on 3GPP narrowband internet of things," IEEE Communications Magazine, vol. 55, no. 3, pp. 117-123, March 2017.

[9] M. Rodrigues and I. Darwazeh, "Fast OFDM: a proposal for doubling the data rate of OFDM schemes," in International conference on Telecommunications, vol. 3, 2002, pp. 484-487.

[10] T. Xu and I. Darwazeh, "Non-orthogonal narrowband Internet of Things: A design for saving bandwidth and doubling the number of connected devices," IEEE Internet of Things Journal, vol. 5, no. 3, pp. 2120-2129, June 2018.

[11] - "Uplink narrowband IoT data rate improvement: Dense modulation formats or non-orthogonal signal waveforms?" in 2018 IEEE 29th Annual International Symposium on Personal, Indoor, and Mobile Radio Communications (PIMRC), Bologna, Italy, Sep. 2018.

[12] _ - "Half-Sinc waveform design for narrowband IoT," in 2018 IEEE 29th Annual International Symposium on Personal, Indoor and Mobile Radio Communications (PIMRC)), Bologna, Italy, Sep. 2018.

[13] X. Wang, P. Ho, and Y. Wu, "Robust channel estimation and ISI cancellation for OFDM systems with suppressed features," IEEE Journal on Selected Areas in Communications, vol. 23, no. 5, pp. 963-972, May 2005.

[14] 3GPP TS 36.213 v.14.2.0, "LTE; evolved universal terrestrial radio access (E-UTRA); physical layer procedures," Rel. 14, Apr. 2017.

[15] S. Isam and I. Darwazeh, "Peak to average power ratio reduction in spectrally efficient FDM systems," in Telecommunications (ICT), 2011 18th International Conference on, May 2011, pp. 363-368.

[16] National Instruments, "USRP-RIO 2953R, Software Defined Radio Reconfigurable Device," http://sine.ni.com/nips/cds/view/p/lang/en/nid/ 213005.

[17] — "Overview of the NI USRP-RIO software defined radio," http://www.ni.com/white-paper/52119/en/, April 2018. 